\title{
Hypokalemic nephropathy in anorexia nervosa
}

\section{Chih-Chia Liang MD, Hung-Chieh Yeh MD}
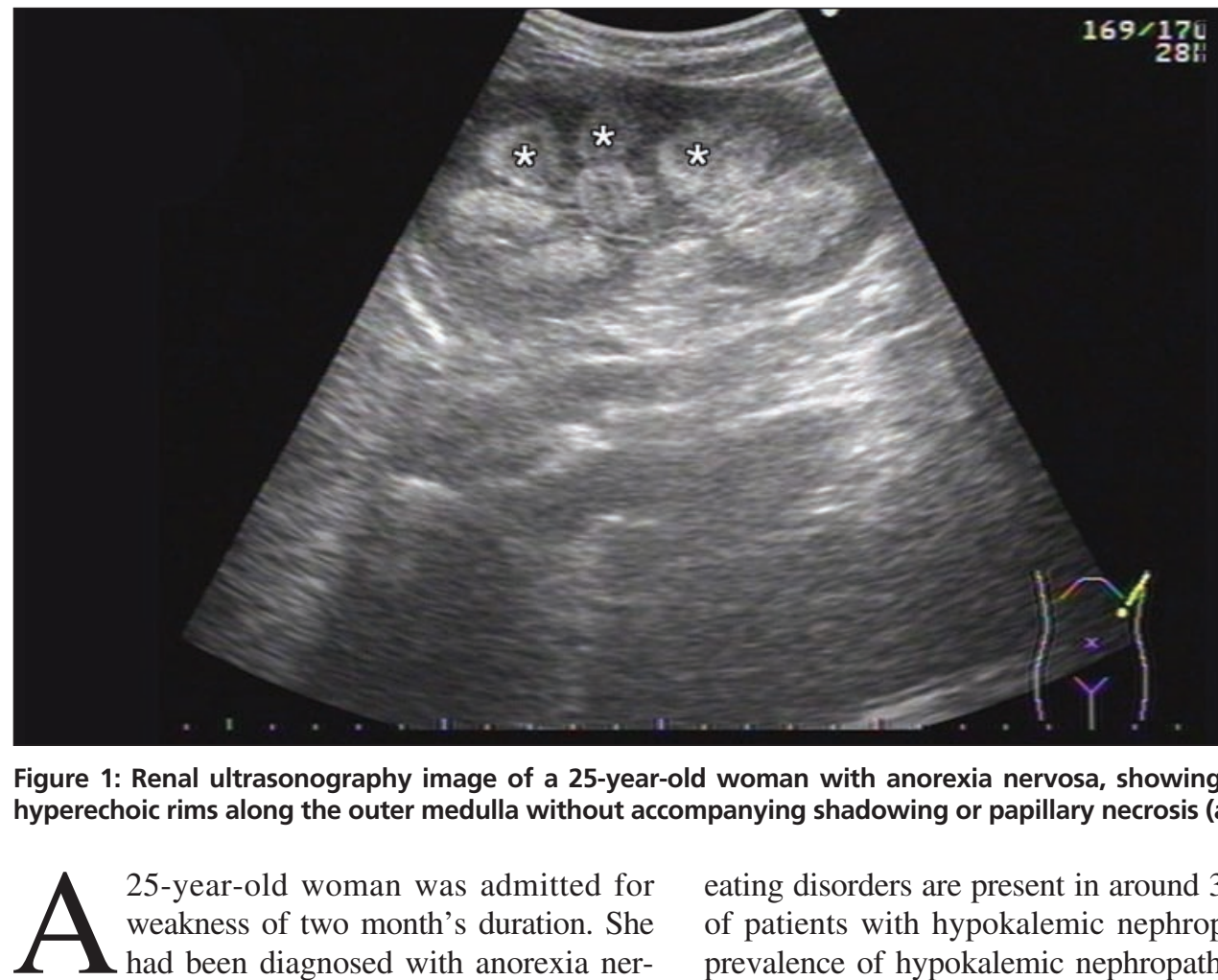
vosa (binge-eating/purging subtype) at the age of 21 years. She had a history of repeated admissions for hypokalemia caused by self-induced vomiting and abuse of laxatives. On examination, her body mass index was $14.3 \mathrm{~kg} / \mathrm{m}^{2}$. Laboratory values showed a serum creatinine level of 51.3 (normal $18-106) \mu \mathrm{mol} / \mathrm{L}$, a serum potassium level of 1.6 (normal $3.5-4.9$ ) $\mathrm{mmol} / \mathrm{L}$, a high urine chloride level of $101.4 \mathrm{mmol} / \mathrm{L}$ and a very low urine calcium level of less then $0.5 \mathrm{mmol} / \mathrm{L}$. After discussion about the laboratory results, the patient admitted to using thiazide before this admission. Renal ultrasonography (Figure 1) showed marked medullary hypertrophy in both kidneys, the hallmark of chronic tubulointerstitial nephritis. ${ }^{1}$

Chronic hypokalemia is commonly caused by purging behaviours or abuse of laxatives or diuretics. It can cause hyperplasia of the renal tubular cells and eventually tubulointerstitial fibrosis and progressive loss of renal function namely, hypokalemic nephropathy. ${ }^{2}$ Although eating disorders are present in around 30\%-40\% of patients with hypokalemic nephropathy, the prevalence of hypokalemic nephropathy in people with eating disorders is unknown. ${ }^{2}$

The level and duration of hypokalemia necessary to develop hypokalemic nephropathy is unclear. Of note, use of diuretics is not a risk factor for hypokalemic nephropathy as long as chronic hypokalemia does not occur. The early stage of hypokalemic nephropathy is usually reversible with potassium replacement, but persistent hypokalemia leading to tubulointerstitial nephritis and associated renal failure may be irreversible. ${ }^{3}$ Mortality in severe instances of eating disorders is more commonly a result of arrhythmia or suicide, rather than a consequence of hypokalemic nephropathy. ${ }^{3}$

\section{References}

1. O'Neill WC. Atlas of renal ultrasonography. 1st ed. Philadelphia (PA): WB Saunders; 2001. p. 57-64.

2. Arimura Y, Tanaka H, Yoshida T, et al. Anorexia nervosa: an important cause of chronic tubulointerstitial nephropathy. Nephrol Dial Transplant 1999;14:957-9.

3. Sim LA, McAlpine DE, Grothe KB, et al. Identification and treatment of eating disorders in the primary care setting. Mayo Clin Proc 2010;85:746-51.
Competing interests: None declared.

This article has been peer reviewed.

Affiliations: From the

Kidney Institute and the

Division of Nephrology, China Medical University Hospital; and the College of Medicine, China Medical

University, Taichung,

Taiwan

Correspondence to: Dr. Hung-Chieh Yeh, hcyeh@pie.com.tw

CMAJ 2011. DOI:10.1503 /cmaj.101790 